\title{
Changes in Sucking Performance from Nonnutritive Sucking to Nutritive Sucking during Breast- and Bottle-Feeding
}

\author{
KATSUMI MIZUNO AND AKI UEDA \\ Division of Neonatology [K.M.], Chiba Children's Hospital, Chiba City, Chiba, Japan 266-0007; Department of Research and \\ Development [A.U.], Pigeon Co. Ltd., Ibaraki, Japan 300-2436
}

\begin{abstract}
Our aim was to obtain a better understanding of the differences between breast-feeding and bottle-feeding, particularly with regard to how sucking performance changes from nonnutritive sucking (NNS) to nutritive sucking (NS). Twenty-two normal term infants were studied while breast-feeding at 4 and $5 \mathrm{~d}$ postpartum. Five of the 22 infants were exclusively breast-fed, but we tested the other 17 infants while breast-feeding and while bottle-feeding. Before the milk ejection reflex (MER) occurs, little milk is available. As such, infants perform NNS before MER. For bottle-feeding, a oneway valve was affixed between the teat and the bottle so that the infants needed to perform NNS until milk flowed into the teat chamber. At the breast, the sucking pressure $(-93.1 \pm 28.3 \mathrm{~mm} \mathrm{Hg})$ was higher during NNS compared with NS $(-77.3 \pm 27.0 \mathrm{~mm} \mathrm{Hg})$. With a bottle, the sucking pressure was lower during NNS $(-27.5 \pm$ $11.2 \mathrm{~mm} \mathrm{Hg})$ compared with NS $(-87.5 \pm 28.5 \mathrm{~mm} \mathrm{Hg})$. Sucking frequency was higher and sucking duration was shorter during NNS compared with that during NS both at the breast and with a bottle. There were significant differences in the changes of sucking pressure and duration from NNS to NS between breast- and bottle-feeding. The change in sucking pressure and duration from NNS to NS differed between breast-feeding and bottle-feeding. Even with a modified bottle and teats, bottle-feeding differs from breast-feeding. (Pediatr Res 59: 728-731, 2006)
\end{abstract}

$\mathrm{O}$ ne of the differences between breast-feeding and bottlefeeding is that infants need to suck nonnutritively at the breast until the MER occurs, because little milk is available before MER (1-3). MER is identified not only by pain or pressure in the breast but also by a change in the sucking pattern of the infant. Inch and Garforth (4) reported that infants demonstrated short, fast bursts of sucking when they attached to the breast. This description is similar to our experience, but, to the best of our knowledge, few objective evaluations of the changes in sucking pattern before and after MER exist. Infants have to suckle the breast to stimulate MER even though they do not obtain milk flow immediately. With MER, milk begins to flow and the infant's sucking pattern changes to a long, slow, continuous pattern. Since the sucking pattern is dependent on the milk flow, it is understandable that infants suck slowly after MER (5). Whether or not infants can suck at the breast effectively and continuously until milk flows

Received August 24, 2005; accepted January 9, 2006

Correspondence: Katsumi Mizuno, M.D., Showa University of Medicine, Department of Pediatrics, 1-5-8 Hatanodai, Shinagawa-ku, Tokyo, Japan 142-8666; e-mail: katsuorobi@aol.com

DOI: 10.1203/01.pdr.0000214993.82214.1c into their mouth is an important factor in successful breastfeeding. The first objective is to evaluate changes in the sucking pattern of breast-feeding infants before and after MER.

With bottle-feeding, an infant obtains milk flow when a teat is inserted into the mouth. There are numerous articles regarding sucking performance with artificial teats during NNS or NS. However, there exist few studies that evaluate how infants change their sucking performance sequentially from NNS to NS. Forty years ago, Wolff (6) examined the NNS and NS patterns of full-term infants and found that sucking frequency is approximately 120 sucks/min during NNS and 60 sucks/min during NS, with infants readily capable of switching from NNS to NS. He also found that the amplitude of sucking in NNS was smaller than that in NS. It should be noted, however, that he measured the pressure generated by the compressing of the lip or tongue, not the intraoral negative pressure.

In this study, we measured intraoral negative pressures during breast- and bottle-feeding and compared them between NNS and NS. We affixed a one-way valve between the teat chamber and the bottle chamber. Therefore, infants were required to suck the teat nonnutritively to obtain milk flow. This situation quite closely resembles sucking at the breast. The second question is whether or not infants change their sucking pattern as they do during breast-feeding, if they must perform NNS to obtain milk flow with the artificial teat. We also compared sucking variables, i.e., sucking pressure, frequency, and duration, during NNS or NS between breast- and bottle-feeding.

\section{SUBJECTS AND METHODS}

Subjects. Normal, term infants were recruited from the local maternity hospital in Chiba prefecture. A total of 25 infants were studied while breast-feeding in the morning at both 4 and $5 \mathrm{~d}$ postpartum. Five of them were exclusively breast-fed so they were not studied while bottle-feeding. The other 20 infants were studied while breast-feeding and also while bottlefeeding with room-temperature expressed breast milk in the afternoon. These infants were fed only by bottle at night. The protocol was approved by the Institutional Review Board of Chiba Children's Hospital. Informed parental consent was obtained if the infants were born after $37 \mathrm{wk}$ gestation; if the Apgar score at 1 and 5 min was higher than 7; if they sucked well at the breast; and if their mothers felt MER while nursing. Before enrollment in the study, two International Board Certified Lactation Consultants (IBCLC) confirmed that the mother's other breast expressed/leaked milk when she felt MER while breast-feeding. In addition, before entry in the study, we confirmed that the

Abbreviations: MER, milk ejection reflex; NNS, non-nutritive sucking; NS, nutritive sucking 
infant's sucking pattern changed before and after the mother's assessment of her MER.

Methods. The interval between the experimental and the preceding sucking ranged between 2 and $4 \mathrm{~h}$. To measure the sucking pressure, a silicone tube of $1 \mathrm{~mm}$ internal diameter was attached to the mother's nipple and emerged inside the infant's mouth. The other end of the tube was connected to a micro-semiconductor pressure transducer (Teac/Kurite transducer, Kurite Co. Ltd., Tokyo, Japan) (Fig. 1). The pressures were amplified using an SA 58 DC amplifier (Teac Co. Ltd., Tokyo, Japan), and were then recorded onto a DR-F3 digital recorder (Teac Co. Ltd.). When we attached the tube to the nipple, we took care to not annoy the infant sucking at the breast with the tube. It is our experience that if there is too long a tube from the top of the nipple head, infants sometimes gag; therefore, we placed the tip of the tube just above the top of the nipple. If infants demonstrated emesis or sucking difficulty, or if the mother felt that infant was sucking differently due to malpositioning of the tubing, we stopped the measurement and postponed it to another feeding session on the same day. When mothers reported sensing MER during the breast-feeding session, we placed a marker on the chart.

With regard to the bottle-feeding session, we fed the same amount of milk previously supplemented $(20-40 \mathrm{~mL})$. The infants were held in a semiupright supine position for feeding, and this position was maintained by one of the investigators throughout the feeding period. The teats, supplied by Pigeon Co. Ltd. (Tokyo, Japan), were adapted to monitor the sucking patterns of the infants and were those used by the studied infants in the nursery. Because the flow rates of artificial teats affect sucking behavior, the teats were standardized; the milk flow rates of the teats were $20 \pm 2 \mathrm{~mL} / 100 \mathrm{~mm}$ $\mathrm{Hg} / \mathrm{min}$, as determined by means of a $100-\mathrm{mm} \mathrm{Hg}$ vacuum pump $(7,8)$. The flow rate is comparable to the system used by Medoff-Cooper et al. (9). The teats were modified to measure the intraoral pressure or suction component. A silicone tube of 1-mm internal diameter was inserted inside the teat and emerged near the teat hole. The other end of the tube was connected to a micro-semiconductor pressure transducer (Teac/Kurite transducer, Kurite Co. Ltd.) (Fig. 2). A one-way valve was affixed between the teat chamber and the bottle chamber so that infants needed to suck the teat nonnutritively until milk flowed into the teat chamber. When the one-way valve was inserted, $-15 \mathrm{~mm}$ $\mathrm{Hg}$ of negative pressure was needed to obtain milk flow.

Sucking variables. The variables were calculated as follows: mean peak negative pressure ( $\mathrm{mm} \mathrm{Hg}$ ), mean sucking frequency (sucks/min), and mean sucking duration of each suck (s) during the continuous sucking phase. We used the average values of two measurements obtained on 4 and $5 \mathrm{~d}$ of age as representative values for the infants.

Statistical analysis. All values were expressed as the mean \pm SD. For the comparison of changes from NNS to NS between breast- and bottle-feeding, we used repeated measures ANOVA with Dr. SPSSII (SPSS Inc., Tokyo, Japan).

The sucking variables and feeding efficiency $(\mathrm{mL} / \mathrm{min})$ were compared between breast- and bottle-feeding either during NNS or NS by using the paired $t$ test. Significance was considered to be $p<0.05$.

\section{RESULTS}

Milk intake and the duration of the breast- and bottlefeedings were as follows: $36.9 \pm 4.5 \mathrm{~mL}$ and $28.5 \pm 4.9 \mathrm{~mL}$,

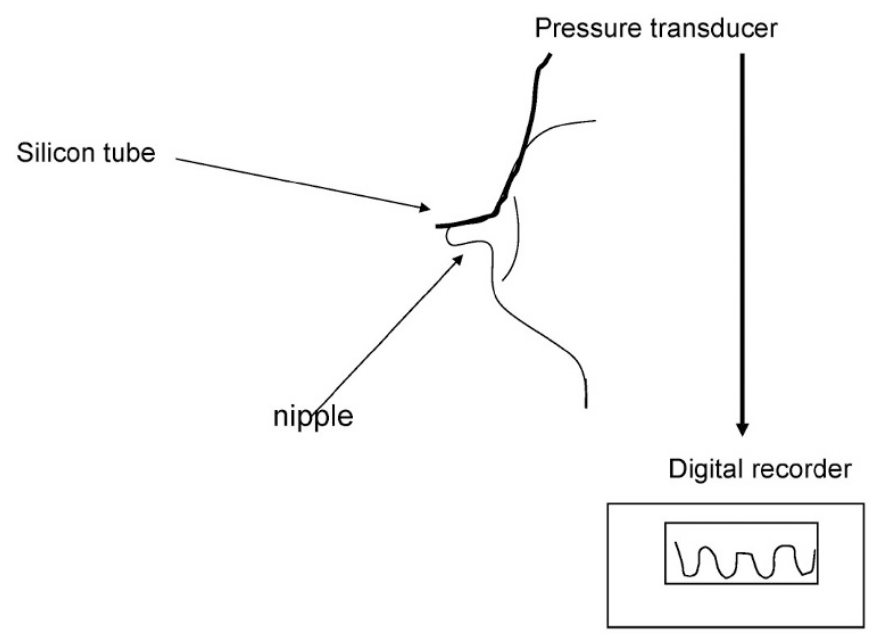

Figure 1. A silicone tube of 1-mm internal diameter was attached to the mother's nipple and emerged inside the infant's mouth. The other end of the tube was connected to a micro-semiconductor pressure transducer.

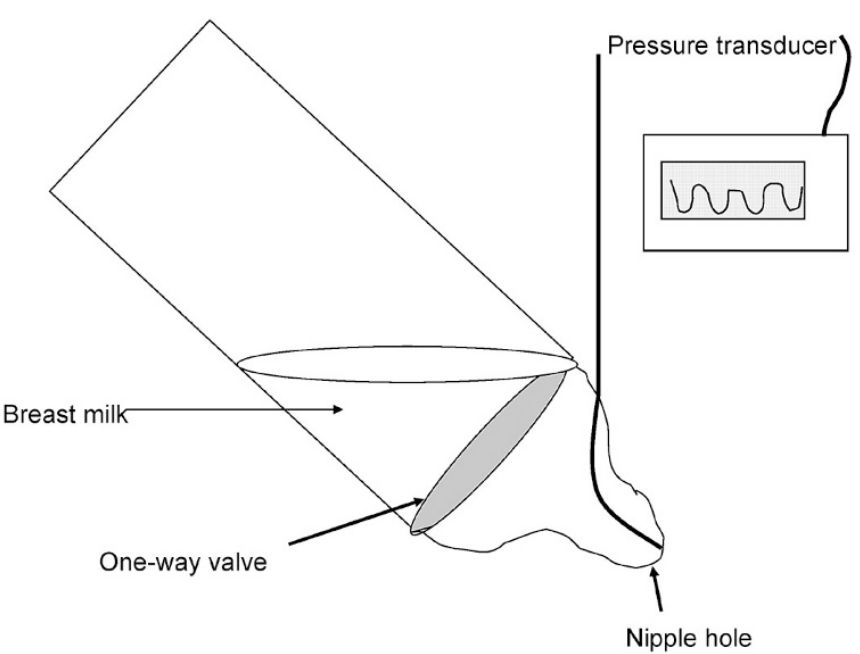

Figure 2. A silicone tube was inserted inside the teat and emerged near the teat hole. The other end of the tube was connected to a micro-semiconductor pressure transducer. A one-way valve was affixed between the teat chamber and the bottle chamber.

and $8.0 \pm 1.5 \mathrm{~min}$ and $4.4 \pm 1.1 \mathrm{~min}$, in breast- and bottlefeeding, respectively. The milk intake during breast-feeding was significantly larger than that during bottle-feeding $(p<0.01)$.

Three infants could not maintain NNS and became fussy before milk flowed into their mouth. These three infants were therefore excluded from the analysis. Measurements from 17 infants ( 9 boys and 8 girls), in which the gestational age was $39.3 \pm 0.9 \mathrm{wk}$ (range, $38.6-41.0 \mathrm{wk}$ ) and the birth weight was $3134 \pm 329 \mathrm{~g}$ (range, 2788-3734 g), were analyzed. Feeding efficiency was $4.7 \pm 0.9 \mathrm{~mL} / \mathrm{min}$ in the breast-feeding session, which is significantly lower than that in the bottle-feeding session $(6.9 \pm 2.0 \mathrm{~mL} / \mathrm{min}, p<0.005)$.

\section{Changes in Sucking Variables from NNS to NS}

Breast-feeding session. Data are shown in Table 1). One infant appeared annoyed while breast-feeding on the first day ( $4 \mathrm{~d}$ postpartum). We measured again at the next feeding session. She did not demonstrate any annoyance during measurement on the second day ( $5 \mathrm{~d}$ postpartum). A total of 17 mother-infant dyads were successfully examined while

Table 1. Suckling parameters (breast-feeding versus bottle-feeding and NNS versus NS)

\begin{tabular}{lccc}
\hline & NNS & NS & $p$ Value \\
\hline Suckling pressure (mm Hg) & & & \\
$\quad$ Breast-feeding & $-97.6 \pm 10.7$ & $-74.5 \pm 6.9$ & $<0.01$ \\
Bottle-feeding & $-27.6 \pm 10.4$ & $-88.6 \pm 26.0$ & $<0.01$ \\
$p$ Value* & $<0.001$ & 0.06 & \\
Suckling frequency (sucks/min) & & & \\
Breast-feeding & $100.8 \pm 10.1$ & $78.2 \pm 7.4$ & $<0.001$ \\
Bottle-feeding & $96.8 \pm 23.6$ & $70.6 \pm 7.8$ & $<0.001$ \\
$p$ Value* & 0.5 & $<0.005$ & \\
Duration of each suck (s) & & & \\
Breast-feeding & $0.49 \pm 0.05$ & $0.64 \pm 0.06$ & $<0.001$ \\
Bottle-feeding & $0.47 \pm 0.09$ & $0.79 \pm 0.08$ & $<0.001$ \\
$p$ Value* & 0.09 & $<0.001$ & \\
\hline
\end{tabular}

These data were obtained during breast-feeding. Values are expressed mean $\pm \mathrm{SD}(n=17)$.

$* p$ Value in comparison between breast-feeding and bottle-feeding. 
breast- and bottle-feeding. Mothers sensed MER at $75.2 \pm$ $12.9 \mathrm{~s}$ after their infants attached to the breast.

The sucking pressure was significantly higher during NNS compared with that during NS $(p<0.01)$. In terms of sucking frequency and duration of each suck, the frequency was significantly higher during NNS $(p<0.001)$ and the duration of each suck was shorter during NNS compared with that during NS ( $p<0.001$, Fig. 3).

Bottle-feeding session. The time taken for milk to flow into the mouth was $59.9 \pm 5.5 \mathrm{~s}$. The sucking pressure was significantly lower during NNS compared with NS $(p<0.01)$. The sucking frequency was significantly higher during NNS compared with NS $(p<0.001)$, and the duration of each suck was shorter during NNS compared with NS $(p<0.001)$ (Fig. 4).

\section{Comparison of Sucking Variables between Breast- and Bottle-Feeding}

Data are shown in Table 1). Sucking pressures during NNS were $-97.6 \pm 10.7$ and $-27.6 \pm 10.4 \mathrm{~mm} \mathrm{Hg}$ and sucking pressures during NS were $-74.5 \pm 6.9$ and $-88.6 \pm 26.0 \mathrm{~mm}$ $\mathrm{Hg}$ while breast- and bottle-feeding, respectively. Sucking pressure during NNS in breast-feeding was significantly higher than that in bottle-feeding $(p<0.001)$, but during NS, pressure did not differ significantly.

Sucking frequencies during NNS were $100.8 \pm 10.1$ and $96.8 \pm 23.6 \mathrm{~mm} \mathrm{Hg}$ and sucking pressures during NS were $78.2 \pm 7.4$ and $70.6 \pm 7.8$ sucks $/ \mathrm{min}$, while breast- and bottle-feeding, respectively. During the NS period, sucking frequency was higher in breast-feeding compared with that in bottle-feeding $(p<0.005)$.

Sucking durations during NNS were $0.49 \pm 0.05$ and 0.47 $\pm 0.09 \mathrm{~s}$ and sucking durations during NS were $0.64 \pm 0.06$ and $0.79 \pm 0.08 \mathrm{~s}$ while breast- and bottle-feeding, respectively. A significant difference was obtained between breastfeeding and bottle-feeding during the NS period $(p<0.001)$.

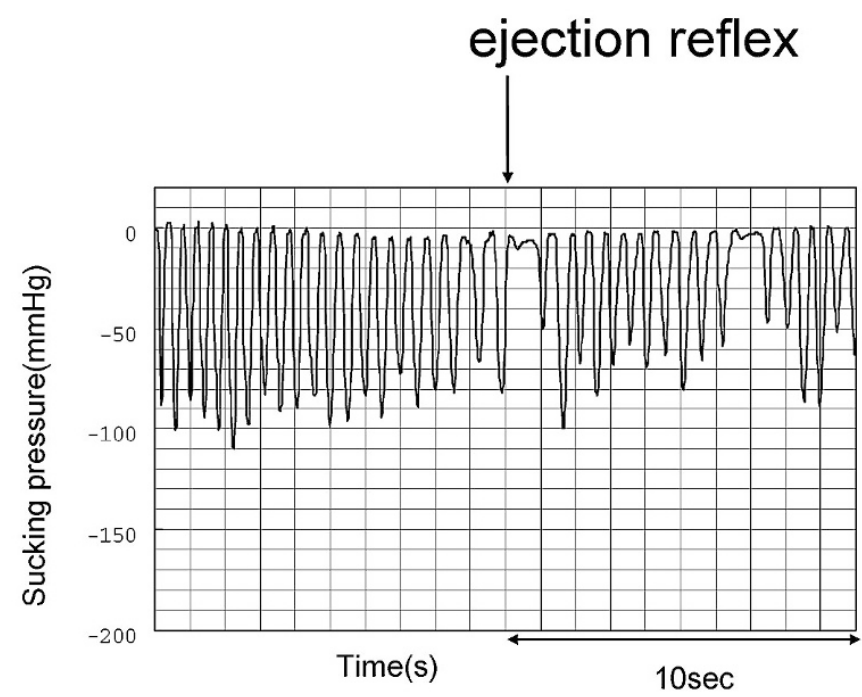

Figure 3. The $x$ axis indicates time and the $y$ axis indicates sucking pressure. This trace was obtained during breast-feeding. After the mother sensed MER, the infant stopped sucking and the sucking pressure lowered thereafter.
Milk flows into mouth

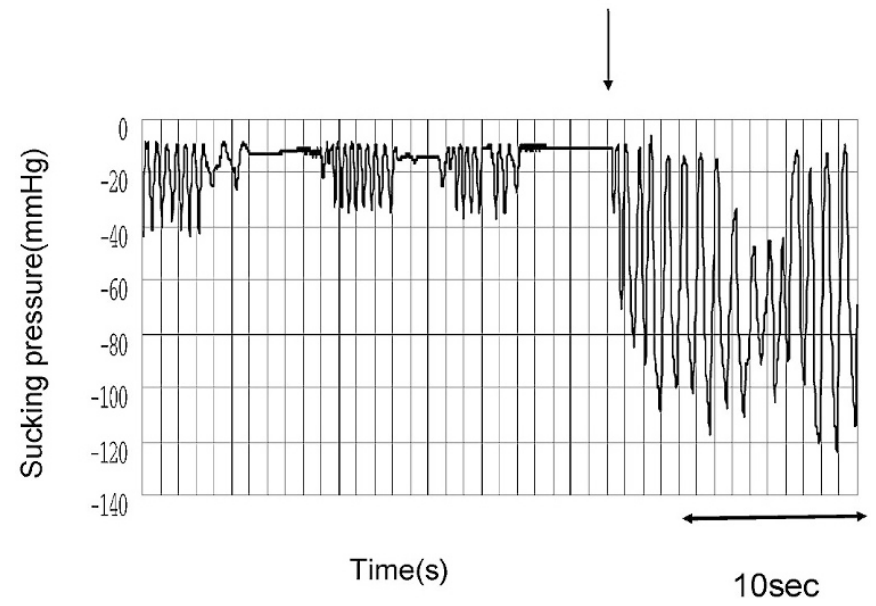

Figure 4. The $x$ axis indicates time and the $y$ axis indicates sucking pressure. This trace was obtained during bottle-feeding. After milk flowed into mouth, the sucking pressure increased.

\section{Comparison of Changes in Sucking Variables from NNS to NS between Breast-Feeding and Bottle-Feeding}

Data are shown in Table 1. The changes in sucking pressure and sucking duration from NNS to NS during breast-feeding was significantly different from those during bottle-feeding $(p$ $<0.01$ for both valuables), although the change in sucking frequency from NNS to NS did not differ between breast- and bottle-feeding.

\section{DISCUSSION}

NNS is commonly observed in breast-feeding infants before MER. NNS could be preparation for NS, although NNS did not have an effect on overall feeding time $(10,11)$. Lau and Schanler (12) recently reported that NNS may not be a good predictor of the NS ability of infants, because infants need to coordinate sucking, swallowing, and breathing while performing NS. Nevertheless, NNS plays an important role in successful breast-feeding for both preterm and term infants in that their NNS stimulates MER. In contrast, with bottlefeeding, milk flows into the infant's mouth as soon as they suck a teat. Therefore, we assumed that this difference could partly explain why sucking performance during breast- and bottle-feeding differs. There have been several articles regarding the differences between breast- and bottle-feeding in terms of tongue movements (13), EMG findings (14), and intraoral pressures (15). Another difference is whether or not infants need to perform NNS to obtain milk. The role of NNS on successful breast-feeding has not yet been fully elucidated. To obtain a better understanding of NNS, we asked whether the insertion of a valve would make bottle-feeding similar to breastfeeding to some extent. However, the results of this study revealed that the feeding performance during bottle- feeding differs from that during breast-feeding, especially in the changes in sucking pressures from NNS to NS. This finding adds another difference between breast- and bottle-feeding.

From the findings of this study, it is evident that while breast-feeding, sucking pressure decreased, sucking duration 
elongated, and sucking frequency decreased from NNS to NS. While breast-feeding, the high sucking rate at the beginning of the feed is believed to stimulate the breast to initiate MER. The slower sucking rate is associated with the infant removing milk from the breast (16-20). Modification of a bottle produced the similar pattern of change in sucking frequency and sucking duration as breast-feeding, although the change in sucking pressure from NNS to NS was opposite to the change during breastfeeding. Medoff-Cooper and Ray (21) compared sucking waves during bottle-feeding between NNS and NS and concluded that NS waves were smoother and more regular than NNS waves; this is also observed in our results. During the NNS period, the duration of the suck cycle was the same for both breast- and bottle-feeding, but during the NS period, the duration was shorter and the frequency was higher for breast-feeding, probably because the milk flow from the artificial teat was greater than that during breast-feeding. The higher feeding efficiency during bottle-feeding compared with that during breast-feeding supports the speculation described above.

Although sucking pressure is similar during the NS period for both the breast and the bottle, as Mathew and Bhatia previously reported (15), the changes in sucking pressure from NNS to NS were opposite between breast- and bottle-feeding. Lau and Kusnierczyk (22) recently compared NNS and NS performance. They applied a finger monitoring device that has two catheters for expression and suction pressures. Although they measured sucking pressures during NNS and NS noncontinuously, infants demonstrated greater sucking pressure during NS compared with that during NNS in their results. The speculation as to why these differences occurred was that artificial teats do not elongate and are more readily compressible by peristaltic tongue movement during feeding than human nipples (23). In addition, because there is no fluid in the teat during NNS, tongue movement could also differ between NNS and NS. In terms of breast-feeding, there is always nipple tissue that is slightly compressible between the tongue and the palate. As such, tongue movement differs from that during bottle-feeding (24). If artificial teats existed that were similar to human nipples in that they were incompressible, pressure alteration from NNS to NS may differ, but currently there are no such artificial teats available

The effect of artificial teats on sucking performance is also an important issue. Because the frequency and duration of sucking during bottle-feeding was lower and longer, respectively, compared with that during breast-feeding, the milk flow could be greater compared with breast-feeding. In terms of the effects of the teat on the experiment, the average sucking pressure obtained in the study corresponded to that of mature infants according to a previous report, that is, $-89 \pm 7.1 \mathrm{~mm} \mathrm{Hg}$ (25). Lau et al. (22) previously reported that mature sucking yields -110 and -160 $\mathrm{mm} \mathrm{Hg}$ of peak negative pressure. In our study, the largest peak negative pressure was within this range and thus it can be said that the teat itself did not have any effect compared with the results previously reported. In addition, because all of the studied infants used the same teat type at the nursery, it was reasonable to also use it for the measurements. The sucking frequency obtained in this study is compatible with values previously published elsewhere (15). In addition, the feeding efficiency in our study was $6.9 \mathrm{~mL} / \mathrm{min}$, that is, infants consume $70 \mathrm{~mL}$ of milk in $10 \mathrm{~min}$, which is usual for bottle-fed infants.

Another issue is whether or not the tubing for measurement during breast-feeding affects sucking performance. The system that we used is similar to the supplemental nursing system, which is currently used elsewhere. Mothers also mentioned that their infants sucked as usual.

In conclusion, infant sucking performance during breastfeeding and during bottle-feeding changed after milk flowed into the mouth. However, the sucking pressure changed in the opposite direction between breast-feeding and bottle-feeding. It is evident from the results of this study that bottle-feeding is a completely different feeding method regardless of attempts to make bottle-feeding more closely resemble breast-feeding.

Acknowledgments. The authors thank Noriko Mizuno, R.N., IBCLC, for her technical support as lactation consultant.

\section{REFERENCES}

1. Mepham TB 1983 Physiological aspects of lactation. In: Mepham TB (ed) Biochemistry of Lactation. Elsevier, New York, page 5.

2. Alekseev NP, Omel'ianuk EV, Talalaeva NE 2000 Dynamics of milk ejection reflex during continuous rhythmic stimulation of the areola-nipple complex of the mammary gland. Ross Fiziol Zh Im I M Sechenoval 86:711-719

3. Ramsay DT, Kent JC, Owens RA, Hartmann PE 2004 Ultrasound imaging of milk ejection in the breast of lactating women. Pediatrics 113:361-367

4. Inch S, Garforth S 1989 Establishing and maintaining breastfeeding. In: Chalmers I, Enkin M, Keirse M (eds) Effective Care in Pregnancy and Childbirth. Oxford University Press, Oxford, UK, pp 1359-1374

5. Mathew OP 1991 Science of bottle feeding. J Pediatr 119:511-519

6. Wolff PH 1968 The serial organization of sucking in the young infant. Pediatrics 42:943-956

7. Mizuno K, Ueda A 2003 The maturation and coordination of sucking, swallowing, and respiration in preterm infants. J Pediatr 142:36-40

8. Mizuno K, Inoue M, Takeuchi T 2000 The effects of body positioning on sucking behavior in sick neonates. Eur J Pediatr 159:827-831

9. Medoff-Cooper B, McGrath JM, Shults J 2002 Feeding patterns of full-term and preterm infants at forty weeks postconceptional age. J Dev Behav Pediatr 23:231236

10. Pickler RH, Frankel HB, Walsh KM, Thompson NM 1996 Effects of nonnutritive sucking on behavioral organization and feeding performance in preterm infants. Nursing Res 45:132-135

11. Pickler RH, Reyna BA 2004 Effects of non-nutritive sucking on nutritive sucking, breathing, and behavior during bottle feedings of preterm infants. Adv Neonatal Care $4: 226-234$

12. Lau C, Schanler RJ 1996 Oral motor function in the neonate. Clin Perinatol 23:161-178

13. Nowak AJ, Smith WL, Erenberg A 1995 Imaging evaluation of breast-feeding and bottle-feeding systems. J Pediatr 126:S130-S134

14. Tamura Y, Matsushita S, Shinoda K, Yoshida S 1996 Development of perioral muscle activity during breast feeding in infants: follow-up study. Pediatr Dent J 6:101-106

15. Mathew OP, Bhatia J 1989 Sucking and breathing patterns during breast- and bottlefeeding in term neonates. Effects of nutrient delivery and composition. Am J Dis Child 143:588-592

16. Luther EC, Arballo JC, Sala NL, Cordero Funes JC 1974 Suckling pressure in humans: relationship to oxytocin-reproducing reflex milk ejection. J Appl Physiol 36:350-353

17. Drewett RF, Woolridge M 1979 Sucking patterns of human babies on the breast. Early Hum Dev 3:315-321

18. Bowen-Jones A, Thompson C, Drewett RF 1982 Milk flow and sucking rates during breast-feeding. Dev Med Child Neurol 24:626-633

19. Woolridge MW 1986 The 'anatomy' of infant sucking. Midwifery 2:164-171

20. Righard L, Alade MO 1992 Sucking technique and its effect on success of breastfeeding. Birth 19:185-189

21. Medoff-Cooper B, Ray W 1995 Neonatal sucking behaviors. Images J Nurs Sch 27:195-200

22. Lau C, Kusnierczyk I 2001 Quantitative evaluation of infant's nonnutritive and nutritive sucking. Dysphagia 16:58-67

23. Nowak AJ, Smith WL, Erenberg A 1995 Imaging evaluation of breast-feeding and bottle-feeding systems. J Pediatr 126:S130-S134

24. Mizuno K, Kani K 2005 Sipping/lapping is a safe alternative feeding method to suckling for preterm infants. Acta Paediatr 94:574-580

25. Mizuno K, Ueda A 2005 Neonatal feeding performance as a predictor of neurodevelopmental outcome at 18 months. Dev Med Child Neurol 47:299-304 